Acta Crystallographica Section C

Crystal Structure

Communications

ISSN 0108-2701

\section{An unusual example of a linearly coordinated acetone ligand in a six-coordinate iron(II) complex}

\author{
Colin A. Kilner and Malcolm A. Halcrow* \\ School of Chemistry, University of Leeds, Woodhouse Lane, Leeds LS2 9JT, England \\ Correspondence e-mail: m.a.halcrow@leeds.ac.uk
}

Received 12 July 2006

Accepted 25 July 2006

Online 31 August 2006

The title compound, acetonediaqua[2,6-bis(3-tert-butylpyrazol-1-yl)pyridine]iron(II) bis(tetrafluoroborate) acetone disolvate, $\quad\left[\mathrm{Fe}\left(\mathrm{C}_{19} \mathrm{H}_{25} \mathrm{~N}_{5}\right)\left(\mathrm{C}_{3} \mathrm{H}_{6} \mathrm{O}\right)\left(\mathrm{H}_{2} \mathrm{O}\right)_{2}\right]\left(\mathrm{BF}_{4}\right)_{2} \cdot 2 \mathrm{C}_{3} \mathrm{H}_{6} \mathrm{O}$, contains a $\mathrm{C}_{2}$-symmetric six-coordinate complex dication, with an acetone ligand in its equatorial plane that is linearly coordinated by symmetry $\left(\mathrm{Fe}-\mathrm{O}=\mathrm{CMe}_{2}=180^{\circ}\right)$. This is a consequence of close steric contacts between the coordinated carbonyl group and the two distal tert-butyl substituents on the tridentate ligand.

\section{Comment}

We are studying the chemistry of homoleptic iron(II) complexes of 2,6-dipyrazolylpyridine and 2,6-dipyrazolylpyrazine derivatives (Halcrow, 2005). Compounds of this type sometimes exhibit an unusual angular Jahn-Teller distortion, in which the metal coordination is severely twisted from its ideal near-octahedral geometry (Holland et al., 2002; Elhaik et al., 2005, 2006; Kilner \& Halcrow, 2006). We have been looking for more examples of this phenomenon and decided to examine the iron(II) chemistry of 2,6-bis(3-tert-butylpyrazol1-yl)pyridine $(L)$ to determine if the bulky ligand substituents might enforce a structural distortion onto an $\left[\mathrm{Fe} L_{2}\right]^{2+}$ centre. In fact, as we had already observed in its copper chemistry (Solanki et al., 2002), $L$ proved too bulky to allow $\left[\mathrm{Fe} L_{2}\right]^{2+}$ to form. Hence, when hydrated $\mathrm{Fe}\left(\mathrm{BF}_{4}\right)_{2}$ and two molar equivalents of $L$ were reacted in $\mathrm{MeOH}, \mathrm{MeCN}$ or $\mathrm{MeNO}_{2}$, the resulting yellow solutions only yielded single crystals of unreacted $L$ (Halcrow, 2005) and/or small amounts of intractable powder when layered with $\mathrm{Et}_{2} \mathrm{O}$. A pure ironcontaining product was only obtained when these reactions were performed in acetone, giving near-colourless crystals of trans-[FeL( $\left.\left(\mathrm{OH}_{2}\right)_{2}\left(\mathrm{OCMe}_{2}\right)\right]\left(\mathrm{BF}_{4}\right)_{2} \cdot 2 \mathrm{Me}_{2} \mathrm{CO}, \quad$ (I). This compound was obtained in pure form when $\mathrm{Fe}\left(\mathrm{BF}_{4}\right)_{2} \cdot 6 \mathrm{H}_{2} \mathrm{O}$ and $L$ were reacted in a 1:1 molar ratio under the same conditions.

The complex dication in (I) spans a crystallographic $C_{2}$ axis passing through atoms Fe1, N2, C5, O16 and C17 (Fig. 1). The bond lengths to Fe1 are in the range expected for a high-spin
iron(II) centre (but see below), while the bond angles are close to those of an ideal octahedron, except for the restricted

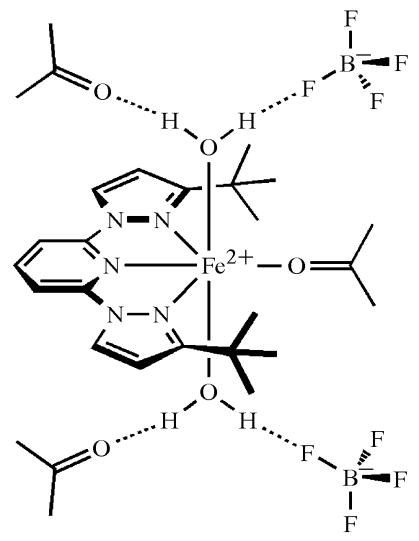

(I)

$74.50(4)^{\circ}$ bite angle of the $L$ ligand (Table 1 ). The metal coordination is completed by an acetone ligand occupying the fourth equatorial coordination site and by two axial water ligands. Notably, the acetone ligand is coordinated in a linear fashion, the $\mathrm{Fe} 1-\mathrm{O} 16-\mathrm{C} 17$ angle being $180^{\circ}$ by symmetry. Linear, rather than bent, coordination of a terminal monodentate dialkyl or alkyl aryl ketone ligand is unusual, particularly in compounds where a significant degree of $d$-orbital metal-ligand covalency might be expected (Fig. 2). The five previous examples with an $\mathrm{Fe}-\mathrm{O}=\mathrm{CR}_{2}$ angle greater than $170^{\circ}$ (Fig. 2) in $d$-block chemistry involve $d^{0} \mathrm{Zr}^{\mathrm{IV}}$ (Sun et al., 1997) or $d^{10} \mathrm{Cu}^{\mathrm{I}}$ (Munakata et al., 1994) and $\mathrm{Hg}^{\mathrm{II}}$ (Lee et al., 2001) metal ions, or weak apical or axial interactions to tetragonal copper(II) centres (Scott \& Holm, 1994; Akitsu \&

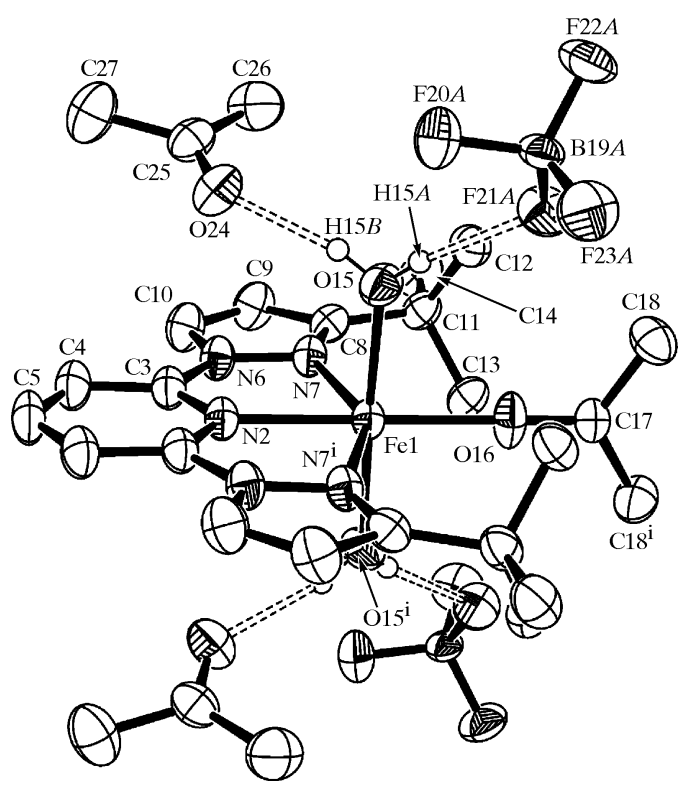

Figure 1

The molecular structure of the components of (I), showing the atomnumbering scheme employed. All C-bound $\mathrm{H}$ atoms, and the minor anion disorder orientation $(\mathrm{B} 19 B / \mathrm{F} 20 B-\mathrm{F} 23 B)$ have been omitted for clarity. Displacement ellipsoids are shown at the $50 \%$ probability level, except for $\mathrm{H}$ atoms, which have arbitrary radii. [Symmetry code: (i) $-x, y,-z+\frac{1}{2}$.] 
Einaga, 2004). Of particular relevance is trans- $\left[\mathrm{RhCl}_{2}\right.$ (Phebox) $\left(\mathrm{OCMe}_{2}\right)$ ] [HPhebox is 1,3-bis(4-methyloxazolin-2yl)benzene], which is isoelectronic with and stereochemically similar to (I) but contains a bent acetone ligand with an $\mathrm{Rh}-$ $\mathrm{O}=\mathrm{C}$ angle of $137(1)^{\circ}$ (Motoyama et al., 2001).

The linear Fe1-O16-C17 angle in (I) is probably sterically imposed by the surrounding tert-butyl groups (Fig. 3), since atom $\mathrm{O} 16$ is in close contact with one $\mathrm{H}$ atom from each of the $\mathrm{C} 12$ and $\mathrm{C} 13$ methyl groups, with $\mathrm{C} 12 \cdots \mathrm{O} 16=3.528$ (3) $\AA$ and $\mathrm{C} 13 \cdots \mathrm{O} 16=3.362$ (3) $\AA$. For comparison, the sum of the van der Waals radii of an $\mathrm{O}$ atom and a methyl group is $3.4 \AA$ (Pauling, 1960). This steric influence is also probably why the

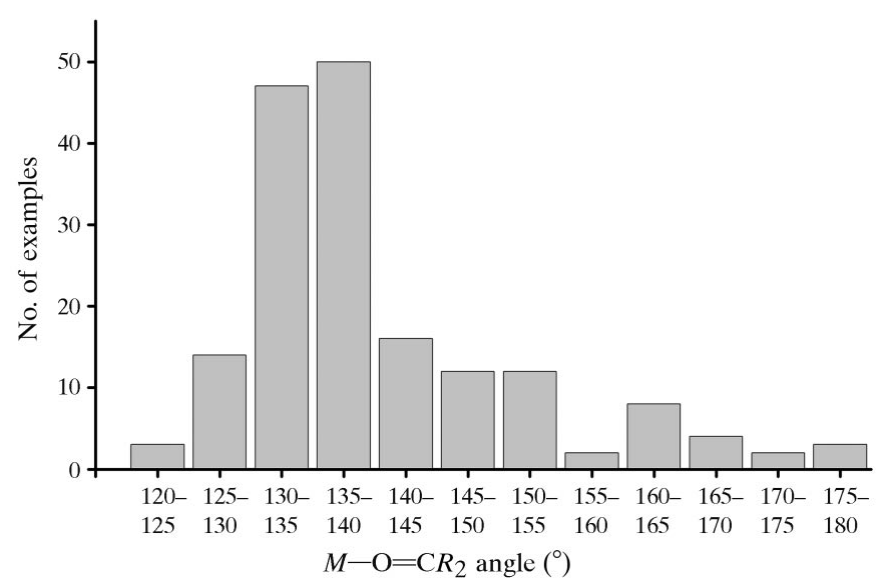

Figure 2

The distribution of $M-\mathrm{O}=\mathrm{C} R_{2}$ angles in $d$ - and $f$-block metal complexes of acetone, and other monodentate dialkyl and alkyl aryl ketones in the Cambridge Structural Database (July 2006 Version; Allen, 2002).

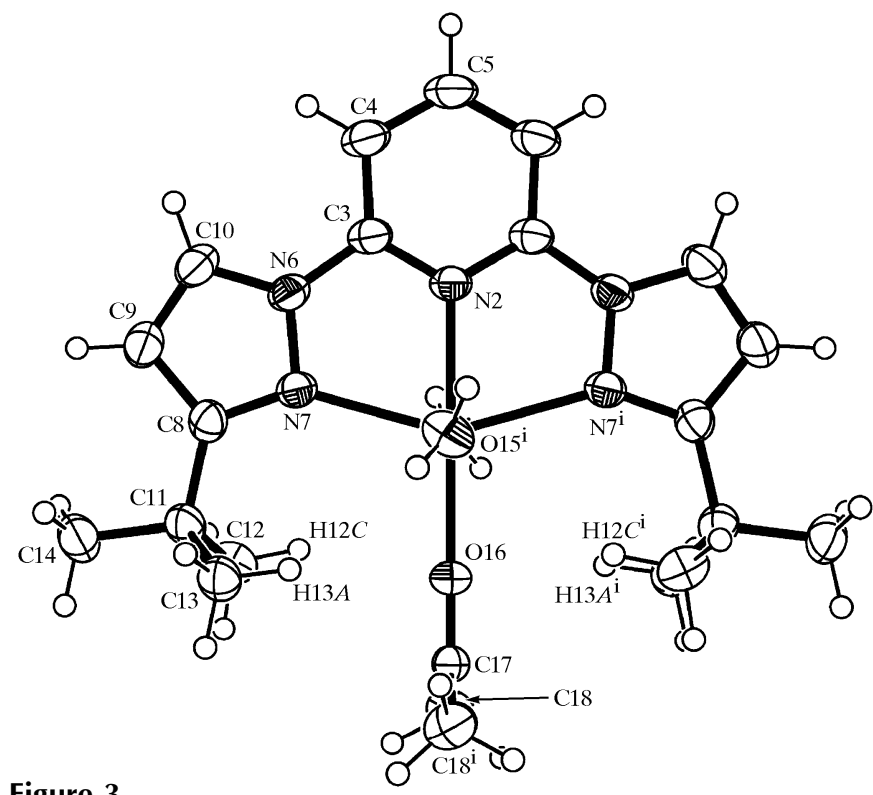

Figure 3

An alternative view of the complex dication in (I), showing the close approach of the tert-butyl methyl groups to the coordinated acetone ligand. Displacement ellipsoids are at the $50 \%$ probability level, except for $\mathrm{H}$ atoms, which have arbitrary radii. The view is approximately parallel to the $\mathrm{O} 15-\mathrm{Fe} 1-\mathrm{O} 15^{\mathrm{i}}$ vector, with $\mathrm{Fe} 1$ and $\mathrm{O} 15$ hidden behind O15. [Symmetry code: (i) $-x, y,-z+\frac{1}{2}$.]
Fe1-N7 bond is $0.07-0.10 \AA$ longer than we have previously observed in high-spin complexes of 2,6-dipyrazolylpyridines with regular six-coordinate structures (Halcrow, 2005, and references therein). In contrast, the $\mathrm{Fe} 1-\mathrm{O} 16$ bond length is somewhat shorter than those in the only other two known high-spin iron(II) acetone complexes, which have $\mathrm{Fe}-\mathrm{O}$ distances of 2.113 (2) $\left[\mathrm{Fe}-\mathrm{O}=\mathrm{CMe}_{2}=134.91(18)^{\circ}\right]$ and 2.123 (2) $\AA$ [135.9 (2) $)^{\circ}$ (Costes et al., 2002). Unfortunately, this sample is too small to determine whether a meaningful correlation exists between the $\mathrm{Fe}-\mathrm{O}$ distance and $\mathrm{Fe}-\mathrm{O}=$ $\mathrm{CR}_{2}$ angle in these complexes. One more iron(II) acetone complex is known (Hamon et al., 1994), but since it contains an intermediate-spin iron centre it is not strictly comparable with (I).

The $\mathrm{O} 15$ water ligand hydrogen bonds to one $\mathrm{F}$ atom of the disordered $\mathrm{BF}_{4}^{-}$anion in the asymmetric unit of (I) through $\mathrm{H} 15 A$, and to the acetone solvent molecule through $\mathrm{H} 15 B$ (Fig. 1 and Table 2). Therefore, (I) exists as discrete [Fe $L$ $\left.\left(\mathrm{OH}_{2}\right)_{2}\left(\mathrm{OCMe}_{2}\right)\right]\left(\mathrm{BF}_{4}\right)_{2} \cdot 2 \mathrm{Me}_{2} \mathrm{CO}$ supramolecules, which associate with each other through van der Waals contacts only.

\section{Experimental}

A solution of $L\left(0.25 \mathrm{~g}, 7.7 \times 10^{-4} \mathrm{~mol}\right)$ (Jameson \& Goldsby, 1990) and $\mathrm{Fe}\left(\mathrm{BF}_{4}\right)_{2} \cdot 6 \mathrm{H}_{2} \mathrm{O}\left(0.13 \mathrm{~g}, 3.9 \times 10^{-4} \mathrm{~mol}\right)$ in acetone $(30 \mathrm{ml})$ was stirred at room temperature in air until all the solid had dissolved. The colourless solution was concentrated in vacuo to $c a 5 \mathrm{ml}$ and filtered. Slow diffusion of diethyl ether vapour into the solution yielded colourless crystals of (I) (yield $0.19 \mathrm{~g}, 64 \%$ ). The crystals decompose to a very pale-green powder following exposure to air for a period of minutes. Analysis of the dried material implied that all the acetone solvent had been lost and about half of the coordinated acetone in the solid had been replaced with water from atmospheric moisture. The stoichiometry of acetone in the final product was confirmed by IR analysis and by the acetone methyl ${ }^{1} \mathrm{H}$ NMR peak listed below, which only integrates to $c a 3.5 \mathrm{H}$ relative to the other peaks in the spectrum [the complex molecule in crystalline (I) would give an acetone peak integrating to $6 \mathrm{H}]$. Analysis found: $\mathrm{C} 38.7, \mathrm{H}$ 5.1, N 10.9\%; calculated for $\left[\mathrm{Fe}\left(\mathrm{C}_{19} \mathrm{H}_{25} \mathrm{~N}_{5}\right)\left(\mathrm{C}_{3} \mathrm{H}_{6} \mathrm{O}\right)_{0.5}\left(\mathrm{H}_{2} \mathrm{O}\right)_{2.5}\right]-$ $\left(\mathrm{BF}_{4}\right)_{2}: \mathrm{C} 39.3, \mathrm{H} 5.3, \mathrm{~N} 11.2 \%$. IR (nujol): $3425(b r, v \mathrm{O}-\mathrm{H}), 1692$ $(m, v \mathrm{C}=\mathrm{O}), 1642(b r, \delta \mathrm{H}-\mathrm{O}-\mathrm{H}), 1070(v s, v \mathrm{~B}-\mathrm{F}) \mathrm{cm}^{-1} \cdot{ }^{1} \mathrm{H}$ NMR $\left(250.1 \mathrm{MHz}, \mathrm{CD}_{3} \mathrm{NO}_{2}, 293 \mathrm{~K}\right.$ ): $\delta$ 69.0, 65.0, 31.9 (all 2H, py

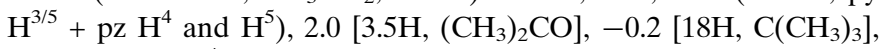
$-2.9\left(1 \mathrm{H}\right.$, py $\left.\mathrm{H}^{4}\right)$.

\section{Crystal data \\ $\left[\mathrm{Fe}\left(\mathrm{C}_{19} \mathrm{H}_{25} \mathrm{~N}_{5}\right)\left(\mathrm{C}_{3} \mathrm{H}_{6} \mathrm{O}\right)\right.$ - $\left.\left(\mathrm{H}_{2} \mathrm{O}\right)_{2}\right]\left(\mathrm{BF}_{4}\right)_{2} \cdot 2 \mathrm{C}_{3} \mathrm{H}_{6} \mathrm{O}$ $M_{r}=763.18$ \\ Monoclinic, $C 2 / c$ \\ $a=24.4575$ (4) ̊ \\ $b=12.7827(1) \AA$ \\ $c=14.5598(4) \AA$ \\ $\beta=126.323(1)^{\circ}$ \\ Data collection \\ Nonius KappaCCD area-detector diffractometer \\ $\omega$ and $\varphi$ scans \\ Absorption correction: multi-scan (SORTAV; Blessing, 1995) \\ $T_{\min }=0.745, T_{\max }=0.981$ \\ (expected range $=0.668-0.879$ )}

$$
\begin{aligned}
& V=3667.40(12) \AA^{3} \\
& Z=4 \\
& D_{x}=1.382 \mathrm{Mg} \mathrm{m}^{-3} \\
& \text { Mo } K \alpha \text { radiation } \\
& \mu=0.49 \mathrm{~mm}^{-1} \\
& T=150 \text { (2) K } \\
& \text { Rectangular prism, colourless } \\
& 0.46 \times 0.33 \times 0.26 \mathrm{~mm}
\end{aligned}
$$

36356 measured reflections 4190 independent reflections 3718 reflections with $I>2 \sigma(I)$ $R_{\text {int }}=0.131$

$\theta_{\text {max }}=27.5^{\circ}$ 


\section{Refinement}

Refinement on $F^{2}$

$R\left[F^{2}>2 \sigma\left(F^{2}\right)\right]=0.046$

$w R\left(F^{2}\right)=0.125$

$S=1.04$

4190 reflections

259 parameters

$\mathrm{H}$ atoms treated by a mixture of independent and constrained refinement

Table 1

Selected geometric parameters $\left(\AA{ }^{\circ}{ }^{\circ}\right)$.

\begin{tabular}{lclr}
\hline $\mathrm{Fe} 1-\mathrm{N} 2$ & $2.112(2)$ & $\mathrm{Fe} 1-\mathrm{O} 15$ & $2.0857(14)$ \\
$\mathrm{Fe} 1-\mathrm{N} 7$ & $2.2739(15)$ & $\mathrm{Fe} 1-\mathrm{O} 16$ & $2.0676(17)$ \\
& & & \\
$\mathrm{N} 2-\mathrm{Fe} 1-\mathrm{N} 7$ & $74.50(4)$ & $\mathrm{N} 7-\mathrm{Fe} 1-\mathrm{O} 15^{\mathrm{i}}$ & $91.31(6)$ \\
$\mathrm{N} 2-\mathrm{Fe} 1-\mathrm{O} 15$ & $91.35(4)$ & $\mathrm{N} 7-\mathrm{Fe} 1-\mathrm{O} 16$ & $105.50(4)$ \\
$\mathrm{N} 2-\mathrm{Fe} 1-\mathrm{O} 16$ & 180 & $\mathrm{O} 15-\mathrm{Fe} 1-\mathrm{O} 15^{\mathrm{i}}$ & $177.30(8)$ \\
$\mathrm{N} 7-\mathrm{Fe} 1-\mathrm{N} 7^{\mathrm{i}}$ & $149.00(7)$ & $\mathrm{O} 15-\mathrm{Fe} 1-\mathrm{O} 16$ & $88.65(4)$ \\
$\mathrm{N} 7-\mathrm{Fe} 1-\mathrm{O} 15$ & $89.41(6)$ & &
\end{tabular}

Symmetry code: (i) $-x, y,-z+\frac{1}{2}$.

Table 2

Hydrogen-bond geometry $\left(\AA,^{\circ}\right)$.

\begin{tabular}{|c|c|c|c|c|}
\hline$D-\mathrm{H} \cdots A$ & $D-\mathrm{H}$ & $\mathrm{H} \cdots A$ & $D \cdots A$ & $D-\mathrm{H} \cdots A$ \\
\hline $\mathrm{O} 15-\mathrm{H} 15 A \cdots \mathrm{F} 21 A$ & $0.80(3)$ & $1.99(3)$ & $2.771(3)$ & $164(3)$ \\
\hline $\mathrm{O} 15-\mathrm{H} 15 A \cdots \mathrm{F} 20 B$ & $0.80(3)$ & $2.01(3)$ & $2.715(5)$ & $147(3)$ \\
\hline $\mathrm{O} 15-\mathrm{H} 15 B \cdots \mathrm{O} 24$ & $0.79(3)$ & $1.92(3)$ & $2.689(2)$ & $165(3)$ \\
\hline
\end{tabular}

The high value of $R_{\text {int }}$ is not a consequence of weak high-angle diffraction, since this parameter does not decrease significantly if only low-angle data are merged. Rather, it may be a consequence of the absorption correction applied, or it may simply reflect the high redundancy in the data collection ( $c a$ nine reflections collected for every unique reflection).

The structure was originally solved and refined in the triclinic space group $P \overline{1}$, with the complex dication lying on a general crystallographic position (i.e. $Z=2$ ). However, a check for higher symmetry following this initial refinement, using the ADDSYM routine in PLATON (Spek, 2003), suggested that the highersymmetry space group $C 2 / c$ might be more appropriate. The data were transformed into $C 2 / c$ and the structure was then solved again and refined, leading to the final model described here.

The asymmetric unit contains half a complex dication, lying on the crystallographic $C_{2}$ axis $\left[0, y, \frac{1}{4}\right]$, which passes through atoms $\mathrm{Fe} 1, \mathrm{~N} 2$, $\mathrm{C} 5, \mathrm{H} 5, \mathrm{O} 16$ and $\mathrm{C} 17$, and one $\mathrm{BF}_{4}{ }^{-}$anion and an acetone solvent molecule lying on general positions. The $\mathrm{BF}_{4}{ }^{-}$ion $\mathrm{B} 19 / \mathrm{F} 20-\mathrm{F} 23$ is disordered over two orientations, labelled $A$ (refined occupancy 0.65 ) and $B(0.35)$. All $\mathrm{B}-\mathrm{F}$ bonds were restrained to 1.38 (2) $\AA$ during refinement, and all F . F F distances within a given disorder orientation to 2.25 (2) $\AA$. All non-H atoms, except for the minor anion disorder site, were refine anisotropically. All C-bound $\mathrm{H}$ atoms were placed in calculated positions and refined using a riding model with the methyl group torsion angles allowed to refine freely $[\mathrm{C}-\mathrm{H}=$ $0.95 \AA$ and $U_{\text {iso }}(\mathrm{H})=1.2 U_{\text {eq }}(\mathrm{C})$ for aryl, and $\mathrm{C}-\mathrm{H}=0.98 \AA$ and $U_{\text {iso }}(\mathrm{H})=1.5 U_{\text {eq }}(\mathrm{C})$ for methyl $\mathrm{H}$ atoms]. The water $\mathrm{H}$ atoms $\mathrm{H} 15 \mathrm{~A}$ and $\mathrm{H} 15 B$ were located in a difference map and allowed to refine freely with a common $U_{\text {iso }}$ parameter, which refined to 0.063 (6) $\AA^{2}$. The refined $\mathrm{O} 15-\mathrm{H}$ distances are 0.79 (3) and 0.80 (3) $\AA$, while the $\mathrm{H} 15 A-\mathrm{O} 15-\mathrm{H} 15 B$ angle is $105(3)^{\circ}$.

Data collection: COLLECT (Nonius, 1999); cell refinement: DENZO-SMN (Otwinowski \& Minor, 1997); data reduction: $D E N Z O-S M N$; program(s) used to solve structure: SHELXS97 (Sheldrick, 1997); program(s) used to refine structure: SHELXL97 (Sheldrick, 1997); molecular graphics: ORTEX (McArdle, 1995); software used to prepare material for publication: local program.

The authors acknowledge the EPSRC and the University of Leeds for funding.

Supplementary data for this paper are available from the IUCr electronic archives (Reference: GG3033). Services for accessing these data are described at the back of the journal.

\section{References}

Akitsu, T. \& Einaga, Y. (2004). Acta Cryst. C60, m162-m164.

Allen, F. H. (2002). Acta Cryst. B58, 380-388.

Blessing, R. H. (1995). Acta Cryst. A51, 33-38.

Costes, J.-P., Clemente-Juan, J. M., Dahan, F., Dumestre, F. \& Tuchagues, J.-P. (2002). Inorg. Chem. 41, 2886-2891.

Elhaïk, J., Evans, D. J., Kilner, C. A. \& Halcrow, M. A. (2005). Dalton Trans. pp. 1693-1700.

Elhaïk, J., Kilner, C. A. \& Halcrow, M. A. (2006). Dalton Trans. pp. 823-830.

Halcrow, M. A. (2005). Coord. Chem. Rev. 249, 2880-2908.

Hamon, P., Toupet, L., Hamon, J. R. \& Lapinte, C. (1994). J. Chem. Soc. Chem. Commun. pp. 931-932.

Holland, J. M., McAllister, J. A., Kilner, C. A., Thornton-Pett, M., Bridgeman, A. J. \& Halcrow, M. A. (2002). J. Chem. Soc. Dalton Trans. pp. 548-554.

Jameson, D. L. \& Goldsby, K. A. (1990). J. Org. Chem. 55, 4992-4994.

Kilner, C. A. \& Halcrow, M. A. (2006). Polyhedron, 25, 235-240.

Lee, H., Knobler, C. B. \& Hawthorne, M. F. (2001). J. Am. Chem. Soc. 123, 8543-8549.

McArdle, P. (1995). J. Appl. Cryst. 28, 65.

Motoyama, Y., Okano, M., Narusawa, H., Makihara, N., Aoki, K. \& Nishiyama, H. (2001). Organometallics, 20, 1580-1591.

Munakata, M., Kuroda-Sowa, T., Maekawa, M., Nakamura, M., Akiyama, S. \& Kitagawa, S. (1994). Inorg. Chem. 33, 1284-1291.

Nonius (1999). COLLECT. Nonius BV, Delft, The Netherlands.

Otwinowski, Z. \& Minor, W. (1997). Methods in Enzymology, Vol. 276, Macromolecular Crystallography, Part A, edited by C. W. Carter Jr \& R. M. Sweet, pp. 307-326. New York: Academic Press.

Pauling, L. (1960). The Nature of the Chemical Bond, 3rd ed., pp. 257-264. Ithaca, New York: Cornell University Press.

Scott, M. J. \& Holm, R. H. (1994). J. Am. Chem. Soc. 116, 11357-11367.

Sheldrick, G. M. (1997). SHELXS97 and SHELXL97. University of Göttingen, Germany.

Solanki, N. K., McInnes, E. J. L., Collison, D., Kilner, C. A., Davies, J. E. \& Halcrow, M. A. (2002). J. Chem. Soc. Dalton Trans. pp. 1625-1630.

Spek, A. L. (2003). J. Appl. Cryst. 36, 7-13.

Sun, Y., Piers, W. E. \& Yap, G. P. A. (1997). Organometallics, 16, 2509-2513. 


\section{supporting information}

Acta Cryst. (2006). C62, m437-m439 [doi:10.1107/S0108270106028903]

\section{An unusual example of a linearly coordinated acetone ligand in a six-coordinate iron(II) complex}

\section{Colin A. Kilner and Malcolm A. Halcrow}

\section{Computing details}

Data collection: COLLECT (Nonius, 1999)); cell refinement: DENZO-SMN (Otwinowski \& Minor, 1997)); data reduction: DENZO-SMN; program(s) used to solve structure: SHELXS97 (Sheldrick, 19971); program(s) used to refine structure: SHELXL97 (Sheldrick, 1997\); molecular graphics: ORTEX (McArdle, 1995〉); software used to prepare material for publication: local program.

diaqua(diethyl ether)[2,6-bis(3-tert-butylpyrazol-1-yl)pyridine]iron(II) bis(tetrafluoroborate) diethyl ether disolvate

Crystal data

$\left[\mathrm{Fe}\left(\mathrm{C}_{19} \mathrm{H}_{25} \mathrm{~N}_{5}\right)\left(\mathrm{C}_{3} \mathrm{H}_{6} \mathrm{O}\right)\left(\mathrm{H}_{2} \mathrm{O}\right)_{2}\right]\left(\mathrm{BF}_{4}\right)_{2} \cdot 2 \mathrm{C}_{3} \mathrm{H}_{6} \mathrm{O}$

$M_{r}=763.18$

Monoclinic $C 2 / c$

Hall symbol: $-\mathrm{C} 2 \mathrm{yc}$

$a=24.4575$ (4) $\AA$

$b=12.7827$ (1) $\AA$

$c=14.5598(4) \AA$

$\beta=126.323(1)^{\circ}$

$V=3667.40(12) \AA^{3}$

$Z=4$

\section{Data collection}

Nonius KappaCCD area-detector diffractometer

Radiation source: fine-focus sealed tube

Graphite monochromator

Detector resolution: 9.091 pixels $\mathrm{mm}^{-1}$

$\omega$ and $\varphi$ scans

Absorption correction: multi-scan

Using multiple and symmetry-related data measurements via the program SORTAV See

R.H. Blessing (1995), Acta Cryst. A51, 33-38

Refinement

Refinement on $F^{2}$

Least-squares matrix: full

$R\left[F^{2}>2 \sigma\left(F^{2}\right)\right]=0.046$

$w R\left(F^{2}\right)=0.125$

$S=1.04$

4190 reflections
$F(000)=1592$

$D_{\mathrm{x}}=1.382 \mathrm{Mg} \mathrm{m}^{-3}$

Mo $K \alpha$ radiation, $\lambda=0.71073 \AA$

Cell parameters from 36356 reflections

$\theta=2.8-27.5^{\circ}$

$\mu=0.49 \mathrm{~mm}^{-1}$

$T=150 \mathrm{~K}$

Rectangular prism, colourless

$0.46 \times 0.33 \times 0.26 \mathrm{~mm}$

$T_{\min }=0.745, T_{\max }=0.981$

36356 measured reflections

4190 independent reflections

3718 reflections with $I>2 \sigma(I)$

$R_{\text {int }}=0.131$

$\theta_{\max }=27.5^{\circ}, \theta_{\min }=2.8^{\circ}$

$h=-31 \rightarrow 31$

$k=-16 \rightarrow 16$

$l=-18 \rightarrow 18$

259 parameters

20 restraints

Primary atom site location: structure-invariant direct methods

Secondary atom site location: difference Fourier map 
Hydrogen site location: inferred from neighbouring sites

$\mathrm{H}$ atoms treated by a mixture of independent and constrained refinement

$w=1 /\left[\sigma^{2}\left(F_{\mathrm{o}}^{2}\right)+(0.062 P)^{2}+2.6971 P\right]$

where $P=\left(F_{\mathrm{o}}^{2}+2 F_{\mathrm{c}}^{2}\right) / 3$

\author{
$(\Delta / \sigma)_{\max }<0.001$ \\ $\Delta \rho_{\max }=0.45$ e $\AA^{-3}$ \\ $\Delta \rho_{\text {min }}=-0.68$ e $\AA^{-3}$ \\ Extinction correction: SHELXL97, \\ $\mathrm{Fc}^{*}=\mathrm{kFc}\left[1+0.001 \times \mathrm{Fc}^{2} \lambda^{3} / \sin (2 \theta)\right]^{-1 / 4}$ \\ Extinction coefficient: 0.0057 (7)
}

\section{Special details}

Experimental. Detector set at $30 \mathrm{~mm}$ from sample with different 2 theta offsets 1 degree phi exposures for chi=0 degree settings 1 degree omega exposures for chi $=90$ degree settings

The high value of $R_{\text {int }}$ reflects the very high redundancy in the data collection ( $c a 9$ reflections collected for every 1 unique).

Geometry. All e.s.d.'s (except the e.s.d. in the dihedral angle between two 1.s. planes) are estimated using the full covariance matrix. The cell e.s.d.'s are taken into account individually in the estimation of e.s.d.'s in distances, angles and torsion angles; correlations between e.s.d.'s in cell parameters are only used when they are defined by crystal symmetry. An approximate (isotropic) treatment of cell e.s.d.'s is used for estimating e.s.d.'s involving l.s. planes.

Refinement. Refinement of $F^{2}$ against ALL reflections. The weighted $R$-factor $w R$ and goodness of fit $S$ are based on $F^{2}$, conventional $R$-factors $R$ are based on $F$, with $F$ set to zero for negative $F^{2}$. The threshold expression of $F^{2}>\sigma\left(F^{2}\right)$ is used only for calculating $R$-factors $(\mathrm{gt})$ etc. and is not relevant to the choice of reflections for refinement. $R$-factors based on $F^{2}$ are statistically about twice as large as those based on $F$, and $R$-factors based on ALL data will be even larger.

The structure was originally solved and refined in the triclinic space group P-1, with the complex dication lying on a general crystallographic position (i.e $Z=2$ ). However, a check for higher symmetry following this initial refinement using the ADSYMM routine in PLATON (Spek, 2003) suggested that the higher symmetry space group $C 2 / c$ might be more appropriate. The data were transformed into $C 2 / c$ and the structure was then re-solved and refined, leading to the final model described here.

The asymmetric unit contains half a complex dication, lying on the crystallographic $\mathrm{C}_{2}$ axis $[0, y, 1 / 4]$, which passes through $\mathrm{Fe} 1, \mathrm{~N} 2, \mathrm{C} 5, \mathrm{H} 5, \mathrm{O} 16$ and $\mathrm{C} 17$, and one $\mathrm{BF}_{4}^{-}$anion and acetone solvent molecule lying on general positions. The $\mathrm{BF}_{4}$ - ion is disordered over two orientations, labelled 'A' (refined occupancy 0.65) and 'B' (0.35). All B-F bonds were restrained to $1.38 \AA$ during refinement, and all $F \cdots F$ distances within a given disorder orientation to 2.25 (2) $\AA$. All non-H atoms except for the minor anion disorder site were refined anisotropically. All C-bound $\mathrm{H}$ atoms were placed in calculated positions and refined using a riding model with the methyl group torsions allowed to refine freely. The water $\mathrm{H}$ atoms were located in the difference map, and allowed to refine freely with a common $U_{\text {eq }}$ thermal parameter. The refined $\mathrm{O} 15-\mathrm{H}$ distances are 0.79 (3) and 0.80 (3) $\AA$, while the $\mathrm{H} 15 \mathrm{~A}-\mathrm{O} 15-\mathrm{H} 15 \mathrm{~B}$ angle is $105(3)^{\circ}$.

Fractional atomic coordinates and isotropic or equivalent isotropic displacement parameters $\left(\AA^{2}\right)$

\begin{tabular}{|c|c|c|c|c|c|}
\hline & $x$ & $y$ & $z$ & $U_{\text {iso }} * / U_{\text {eq }}$ & Occ. $(<1)$ \\
\hline $\mathrm{Fe} 1$ & 0.0000 & $0.30658(2)$ & 0.2500 & $0.02901(14)$ & \\
\hline $\mathrm{N} 2$ & 0.0000 & $0.47179(15)$ & 0.2500 & $0.0323(4)$ & \\
\hline C3 & $0.02961(9)$ & $0.52457(13)$ & $0.21176(14)$ & $0.0346(4)$ & \\
\hline $\mathrm{C} 4$ & $0.03140(10)$ & $0.63327(14)$ & $0.20994(17)$ & $0.0431(4)$ & \\
\hline $\mathrm{H} 4$ & 0.0531 & 0.6695 & 0.1826 & $0.052 *$ & \\
\hline $\mathrm{C} 5$ & 0.0000 & $0.68583(19)$ & 0.2500 & $0.0467(7)$ & \\
\hline H5 & 0.0000 & 0.7601 & 0.2500 & $0.056^{*}$ & \\
\hline N6 & $0.05990(8)$ & $0.46157(11)$ & $0.17439(13)$ & 0.0355 & \\
\hline N7 & $0.06095(7)$ & $0.35412(11)$ & $0.18478(12)$ & $0.0326(3)$ & \\
\hline $\mathrm{C} 8$ & $0.09541(9)$ & 0.31925 (14) & $0.14600(15)$ & $0.0340(4)$ & \\
\hline C9 & $0.11584(11)$ & $0.40403(16)$ & $0.11037(18)$ & $0.0455(5)$ & \\
\hline H9 & 0.1406 & 0.4002 & 0.0791 & $0.055^{*}$ & \\
\hline $\mathrm{C} 10$ & $0.09313(11)$ & $0.49174(15)$ & $0.12972(18)$ & $0.0449(5)$ & \\
\hline H10 & 0.0992 & 0.5615 & 0.1149 & $0.054 *$ & \\
\hline $\mathrm{C} 11$ & $0.10866(9)$ & $0.20394(14)$ & $0.14358(16)$ & $0.0363(4)$ & \\
\hline
\end{tabular}




\begin{tabular}{|c|c|c|c|c|c|}
\hline $\mathrm{C} 12$ & $0.14913(10)$ & $0.15894(16)$ & $0.26494(17)$ & $0.0437(4)$ & \\
\hline H12A & 0.1943 & 0.1911 & 0.3115 & $0.066^{*}$ & \\
\hline H12B & 0.1538 & 0.0831 & 0.2621 & $0.066^{*}$ & \\
\hline $\mathrm{H} 12 \mathrm{C}$ & 0.1252 & 0.1739 & 0.2986 & $0.066^{*}$ & \\
\hline $\mathrm{C} 13$ & $0.04054(9)$ & $0.14670(15)$ & $0.06605(16)$ & $0.0403(4)$ & \\
\hline $\mathrm{H} 13 \mathrm{~A}$ & 0.0136 & 0.1568 & 0.0952 & $0.060 *$ & \\
\hline H13B & 0.0488 & 0.0718 & 0.0649 & $0.060 *$ & \\
\hline $\mathrm{H} 13 \mathrm{C}$ & 0.0157 & 0.1749 & -0.0115 & $0.060^{*}$ & \\
\hline $\mathrm{C} 14$ & $0.14970(11)$ & $0.18863(17)$ & $0.0962(2)$ & $0.0484(5)$ & \\
\hline H14A & 0.1246 & 0.2181 & 0.0191 & $0.073 *$ & \\
\hline H14B & 0.1572 & 0.1138 & 0.0933 & $0.073^{*}$ & \\
\hline $\mathrm{H} 14 \mathrm{C}$ & 0.1935 & 0.2242 & 0.1456 & $0.073^{*}$ & \\
\hline $\mathrm{O} 15$ & $0.08949(7)$ & $0.30274(11)$ & $0.41547(12)$ & $0.0420(3)$ & \\
\hline $\mathrm{H} 15 \mathrm{~A}$ & $0.1007(14)$ & $0.267(2)$ & $0.469(3)$ & $0.063(6)^{*}$ & \\
\hline H15B & $0.1146(15)$ & $0.351(2)$ & $0.441(2)$ & $0.063(6)^{*}$ & \\
\hline O16 & 0.0000 & $0.14483(13)$ & 0.2500 & $0.0447(5)$ & \\
\hline $\mathrm{C} 17$ & 0.0000 & $0.04961(18)$ & 0.2500 & $0.0322(5)$ & \\
\hline $\mathrm{C} 18$ & $0.05461(11)$ & $-0.01097(17)$ & $0.34977(16)$ & $0.0470(5)$ & \\
\hline H18A & 0.0818 & -0.0469 & 0.3302 & $0.071^{*}$ & \\
\hline H18B & 0.0344 & -0.0627 & 0.3712 & $0.071^{*}$ & \\
\hline $\mathrm{H} 18 \mathrm{C}$ & 0.0837 & 0.0366 & 0.4139 & $0.071^{*}$ & \\
\hline B19A & $0.1530(4)$ & $0.1692(5)$ & $0.6886(5)$ & $0.0332(14)$ & 0.65 \\
\hline F20A & $0.16075(12)$ & $0.27801(15)$ & $0.6986(2)$ & $0.0612(6)$ & 0.65 \\
\hline F21A & $0.12620(10)$ & $0.14616(16)$ & $0.57387(14)$ & $0.0493(4)$ & 0.65 \\
\hline $\mathrm{F} 22 \mathrm{~A}$ & $0.21371(10)$ & $0.1205(2)$ & 0.76035 (19) & $0.0547(6)$ & 0.65 \\
\hline F23A & $0.1030(2)$ & $0.1382(4)$ & $0.7047(4)$ & $0.0624(9)$ & 0.65 \\
\hline B19B & $0.1408(6)$ & $0.1662(10)$ & $0.6793(11)$ & $0.037(4)^{*}$ & 0.35 \\
\hline F20B & $0.1244(2)$ & $0.2604(3)$ & $0.6267(4)$ & $0.0604(10)^{*}$ & 0.35 \\
\hline F21B & $0.1284(2)$ & $0.0865(4)$ & $0.6080(4)$ & $0.0618(10)^{*}$ & 0.35 \\
\hline F22B & $0.2102(4)$ & $0.1638(5)$ & $0.7691(6)$ & $0.086(2)^{*}$ & 0.35 \\
\hline F23B & $0.1117(4)$ & $0.1530(7)$ & $0.7312(6)$ & $0.056(2)^{*}$ & 0.35 \\
\hline $\mathrm{O} 24$ & $0.16066(8)$ & $0.47987(12)$ & $0.46096(14)$ & $0.0504(4)$ & \\
\hline $\mathrm{C} 25$ & $0.20620(10)$ & $0.50368(16)$ & $0.45374(16)$ & $0.0423(4)$ & \\
\hline $\mathrm{C} 26$ & $0.24263(12)$ & $0.4236(2)$ & $0.4346(2)$ & $0.0601(6)$ & \\
\hline $\mathrm{H} 26 \mathrm{~A}$ & 0.2232 & 0.3544 & 0.4274 & $0.090^{*}$ & \\
\hline H26B & 0.2908 & 0.4235 & 0.4992 & $0.090^{*}$ & \\
\hline $\mathrm{H} 26 \mathrm{C}$ & 0.2379 & 0.4400 & 0.3644 & $0.090^{*}$ & \\
\hline $\mathrm{C} 27$ & $0.22717(14)$ & $0.61492(19)$ & $0.4639(2)$ & $0.0624(6)$ & \\
\hline H27A & 0.1976 & 0.6596 & 0.4717 & $0.094 *$ & \\
\hline H27B & 0.2235 & 0.6352 & 0.3955 & $0.094 *$ & \\
\hline $\mathrm{H} 27 \mathrm{C}$ & 0.2743 & 0.6231 & 0.5312 & $0.094 *$ & \\
\hline
\end{tabular}

Atomic displacement parameters $\left(\AA^{2}\right)$

\begin{tabular}{lllllll}
\hline & $U^{11}$ & $U^{22}$ & $U^{33}$ & $U^{12}$ & $U^{13}$ & $U^{23}$ \\
\hline $\mathrm{Fe} 1$ & $0.0367(2)$ & $0.0227(2)$ & $0.0289(2)$ & 0.000 & $0.02004(16)$ & 0.000 \\
$\mathrm{~N} 2$ & $0.0415(10)$ & $0.0238(9)$ & $0.0314(10)$ & 0.000 & $0.0215(9)$ & 0.000 \\
$\mathrm{C} 3$ & $0.0431(9)$ & $0.0262(8)$ & $0.0324(8)$ & $-0.0010(7)$ & $0.0212(7)$ & $0.0009(6)$
\end{tabular}


supporting information

\begin{tabular}{lllllll} 
C4 & $0.0562(11)$ & $0.0278(8)$ & $0.0461(10)$ & $-0.0026(8)$ & $0.0308(9)$ & $0.0034(7)$ \\
C5 & $0.0636(18)$ & $0.0226(11)$ & $0.0525(16)$ & 0.000 & $0.0337(15)$ & 0.000 \\
N6 & $0.0468(8)$ & $0.0280(7)$ & $0.0361(7)$ & $-0.0025(6)$ & $0.0270(7)$ & $0.0023(6)$ \\
N7 & $0.0411(7)$ & $0.0264(7)$ & $0.0319(7)$ & $-0.0021(6)$ & $0.0225(6)$ & $-0.0003(5)$ \\
C8 & $0.0364(8)$ & $0.0374(9)$ & $0.0304(8)$ & $-0.0050(7)$ & $0.0209(7)$ & $-0.0047(6)$ \\
C9 & $0.0569(11)$ & $0.0441(10)$ & $0.0511(11)$ & $-0.0087(9)$ & $0.0406(10)$ & $-0.0041(8)$ \\
C10 & $0.0585(11)$ & $0.0381(10)$ & $0.0490(11)$ & $-0.0066(8)$ & $0.0378(10)$ & $0.0039(8)$ \\
C11 & $0.0371(9)$ & $0.0365(9)$ & $0.0379(9)$ & $-0.0057(7)$ & $0.0237(8)$ & $-0.0091(7)$ \\
C12 & $0.0444(10)$ & $0.0385(10)$ & $0.0423(10)$ & $0.0034(8)$ & $0.0225(8)$ & $-0.0035(8)$ \\
C13 & $0.0417(9)$ & $0.0408(10)$ & $0.0417(10)$ & $-0.0085(8)$ & $0.0265(8)$ & $-0.0108(8)$ \\
C14 & $0.0488(11)$ & $0.0503(12)$ & $0.0591(13)$ & $-0.0105(9)$ & $0.0392(10)$ & $-0.0180(9)$ \\
O15 & $0.0429(7)$ & $0.0415(8)$ & $0.0318(7)$ & $-0.0085(6)$ & $0.0168(6)$ & $0.0014(5)$ \\
O16 & $0.0665(12)$ & $0.0229(8)$ & $0.0677(13)$ & 0.000 & $0.0523(11)$ & 0.000 \\
C17 & $0.0425(12)$ & $0.0274(11)$ & $0.0337(11)$ & 0.000 & $0.0264(10)$ & 0.000 \\
C18 & $0.0538(11)$ & $0.0454(11)$ & $0.0348(9)$ & $0.0091(9)$ & $0.0223(9)$ & $0.0031(8)$ \\
B19A & $0.026(2)$ & $0.037(2)$ & $0.023(2)$ & $0.0050(17)$ & $0.0072(17)$ & $0.0005(12)$ \\
F20A & $0.0697(13)$ & $0.0347(10)$ & $0.0724(15)$ & $-0.0012(9)$ & $0.0383(12)$ & $-0.0085(9)$ \\
F21A & $0.0558(11)$ & $0.0558(11)$ & $0.0305(8)$ & $0.0039(9)$ & $0.0224(8)$ & $-0.0028(8)$ \\
F22A & $0.0386(10)$ & $0.0605(14)$ & $0.0410(10)$ & $0.0170(10)$ & $0.0103(8)$ & $0.0088(10)$ \\
F23A & $0.0652(18)$ & $0.073(2)$ & $0.0506(19)$ & $-0.0043(15)$ & $0.0353(17)$ & $0.0109(18)$ \\
O24 & $0.0524(8)$ & $0.0494(8)$ & $0.0563(9)$ & $-0.0147(6)$ & $0.0360(7)$ & $-0.0127(7)$ \\
C25 & $0.0435(9)$ & $0.0481(11)$ & $0.0322(9)$ & $-0.0088(8)$ & $0.0208(8)$ & $-0.0073(7)$ \\
C26 & $0.0542(12)$ & $0.0686(15)$ & $0.0563(13)$ & $0.0015(11)$ & $0.0320(11)$ & $-0.0099(11)$ \\
C27 & $0.0763(16)$ & $0.0543(13)$ & $0.0762(16)$ & $-0.0187(12)$ & $0.0559(14)$ & $-0.0118(12)$ \\
& & & & & & \\
\hline
\end{tabular}

Geometric parameters $\left(\AA,{ }^{\circ}\right)$

\begin{tabular}{llll}
\hline $\mathrm{Fe} 1-\mathrm{N} 2$ & $2.112(2)$ & $\mathrm{C} 14-\mathrm{H} 14 \mathrm{~A}$ & 0.9800 \\
$\mathrm{Fe} 1-\mathrm{N} 7$ & $2.2739(15)$ & $\mathrm{C} 14-\mathrm{H} 14 \mathrm{~B}$ & 0.9800 \\
$\mathrm{Fe} 1-\mathrm{O} 15$ & $2.0857(14)$ & $\mathrm{C} 14-\mathrm{H} 14 \mathrm{C}$ & 0.9800 \\
$\mathrm{Fe} 1-\mathrm{O} 16$ & $2.0676(17)$ & $\mathrm{O} 15-\mathrm{H} 15 \mathrm{~A}$ & $0.80(3)$ \\
$\mathrm{N} 2-\mathrm{C} 3$ & $1.329(2)$ & $\mathrm{O} 15-\mathrm{H} 15 \mathrm{~B}$ & $0.79(3)$ \\
$\mathrm{C} 3-\mathrm{C} 4$ & $1.391(2)$ & $\mathrm{O} 16-\mathrm{C} 17$ & $1.217(3)$ \\
$\mathrm{C} 3-\mathrm{N} 6$ & $1.404(2)$ & $\mathrm{C} 17-\mathrm{C} 18$ & $1.482(2)$ \\
$\mathrm{C} 4-\mathrm{C} 5$ & $1.383(2)$ & $\mathrm{C} 18-\mathrm{H} 18 \mathrm{~A}$ & 0.9800 \\
$\mathrm{C} 4-\mathrm{H} 4$ & 0.9500 & $\mathrm{C} 18-\mathrm{H} 18 \mathrm{~B}$ & 0.9800 \\
$\mathrm{C} 5-\mathrm{H} 5$ & 0.9500 & $\mathrm{C} 18-\mathrm{H} 18 \mathrm{C}$ & 0.9800 \\
$\mathrm{~N} 6-\mathrm{C} 10$ & $1.363(2)$ & $\mathrm{B} 19 \mathrm{~A}-\mathrm{F} 22 \mathrm{~A}$ & $1.358(6)$ \\
$\mathrm{N} 6-\mathrm{N} 7$ & $1.3803(19)$ & $\mathrm{B} 19 \mathrm{~A}-\mathrm{F} 20 \mathrm{~A}$ & $1.400(6)$ \\
$\mathrm{N} 7-\mathrm{C} 8$ & $1.338(2)$ & $\mathrm{B} 19 \mathrm{~A}-\mathrm{F} 21 \mathrm{~A}$ & $1.418(6)$ \\
$\mathrm{C} 8-\mathrm{C} 9$ & $1.414(3)$ & $\mathrm{B} 19 \mathrm{~A}-\mathrm{F} 23 \mathrm{~A}$ & $1.431(8)$ \\
$\mathrm{C} 8-\mathrm{C} 11$ & $\mathrm{~B} 19 \mathrm{~B}-\mathrm{F} 23 \mathrm{~B}$ & $1.322(14)$ \\
$\mathrm{C} 9-\mathrm{C} 10$ & $1.514(2)$ & $\mathrm{B} 19 \mathrm{~B}-\mathrm{F} 21 \mathrm{~B}$ & $1.355(13)$ \\
$\mathrm{C} 9-\mathrm{H} 9$ & $1.353(3)$ & $\mathrm{B} 19 \mathrm{~B}-\mathrm{F} 20 \mathrm{~B}$ & $1.355(13)$ \\
$\mathrm{C} 10-\mathrm{H} 10$ & 0.9500 & $\mathrm{~B} 19 \mathrm{~B}-\mathrm{F} 22 \mathrm{~B}$ & $1.400(12)$ \\
$\mathrm{C} 11-\mathrm{C} 14$ & 0.9500 & $\mathrm{O} 24-\mathrm{C} 25$ & $1.218(2)$ \\
$\mathrm{C} 11-\mathrm{C} 13$ & $1.531(3)$ & $\mathrm{C} 25-\mathrm{C} 26$ & $1.488(3)$ \\
$\mathrm{C} 11-\mathrm{C} 12$ & $1.535(2)$ & $\mathrm{C} 25-\mathrm{C} 27$ & $1.489(3)$
\end{tabular}




\begin{tabular}{|c|c|c|c|}
\hline $\mathrm{C} 12-\mathrm{H} 12 \mathrm{~A}$ & 0.9800 & $\mathrm{C} 26-\mathrm{H} 26 \mathrm{~A}$ & 0.9800 \\
\hline $\mathrm{C} 12-\mathrm{H} 12 \mathrm{~B}$ & 0.9800 & $\mathrm{C} 26-\mathrm{H} 26 \mathrm{~B}$ & 0.9800 \\
\hline $\mathrm{C} 12-\mathrm{H} 12 \mathrm{C}$ & 0.9800 & $\mathrm{C} 26-\mathrm{H} 26 \mathrm{C}$ & 0.9800 \\
\hline $\mathrm{C} 13-\mathrm{H} 13 \mathrm{~A}$ & 0.9800 & $\mathrm{C} 27-\mathrm{H} 27 \mathrm{~A}$ & 0.9800 \\
\hline $\mathrm{C} 13-\mathrm{H} 13 \mathrm{~B}$ & 0.9800 & $\mathrm{C} 27-\mathrm{H} 27 \mathrm{~B}$ & 0.9800 \\
\hline $\mathrm{C} 13-\mathrm{H} 13 \mathrm{C}$ & 0.9800 & $\mathrm{C} 27-\mathrm{H} 27 \mathrm{C}$ & 0.9800 \\
\hline $\mathrm{N} 2-\mathrm{Fe} 1-\mathrm{N} 7$ & $74.50(4)$ & $\mathrm{C} 11-\mathrm{C} 13-\mathrm{H} 13 \mathrm{C}$ & 109.5 \\
\hline $\mathrm{N} 2-\mathrm{Fe} 1-\mathrm{O} 15$ & $91.35(4)$ & $\mathrm{H} 13 \mathrm{~A}-\mathrm{C} 13-\mathrm{H} 13 \mathrm{C}$ & 109.5 \\
\hline $\mathrm{N} 2-\mathrm{Fe} 1-\mathrm{O} 16$ & 180 & $\mathrm{H} 13 \mathrm{~B}-\mathrm{C} 13-\mathrm{H} 13 \mathrm{C}$ & 109.5 \\
\hline $\mathrm{N} 7-\mathrm{Fe} 1-\mathrm{N} 7^{\mathrm{i}}$ & $149.00(7)$ & $\mathrm{C} 11-\mathrm{C} 14-\mathrm{H} 14 \mathrm{~A}$ & 109.5 \\
\hline $\mathrm{N} 7-\mathrm{Fe} 1-\mathrm{O} 15$ & $89.41(6)$ & $\mathrm{C} 11-\mathrm{C} 14-\mathrm{H} 14 \mathrm{~B}$ & 109.5 \\
\hline $\mathrm{N} 7-\mathrm{Fe} 1-\mathrm{O} 15^{\mathrm{i}}$ & $91.31(6)$ & $\mathrm{H} 14 \mathrm{~A}-\mathrm{C} 14-\mathrm{H} 14 \mathrm{~B}$ & 109.5 \\
\hline $\mathrm{N} 7-\mathrm{Fe} 1-\mathrm{O} 16$ & $105.50(4)$ & $\mathrm{C} 11-\mathrm{C} 14-\mathrm{H} 14 \mathrm{C}$ & 109.5 \\
\hline $\mathrm{O} 15-\mathrm{Fe} 1-\mathrm{O} 15^{\mathrm{i}}$ & $177.30(8)$ & $\mathrm{H} 14 \mathrm{~A}-\mathrm{C} 14-\mathrm{H} 14 \mathrm{C}$ & 109.5 \\
\hline $\mathrm{O} 15-\mathrm{Fe} 1-\mathrm{O} 16$ & $88.65(4)$ & $\mathrm{H} 14 \mathrm{~B}-\mathrm{C} 14-\mathrm{H} 14 \mathrm{C}$ & 109.5 \\
\hline $\mathrm{C} 33^{\mathrm{i}-\mathrm{N}} 2-\mathrm{C} 3$ & $119.0(2)$ & $\mathrm{Fe} 1-\mathrm{O} 15-\mathrm{H} 15 \mathrm{~A}$ & $131(2)$ \\
\hline $\mathrm{C} 3-\mathrm{N} 2-\mathrm{Fe} 1$ & $120.50(10)$ & $\mathrm{Fe} 1-\mathrm{O} 15-\mathrm{H} 15 \mathrm{~B}$ & $121(2)$ \\
\hline $\mathrm{N} 2-\mathrm{C} 3-\mathrm{C} 4$ & $123.10(18)$ & $\mathrm{H} 15 \mathrm{~A}-\mathrm{O} 15-\mathrm{H} 15 \mathrm{~B}$ & $105(3)$ \\
\hline $\mathrm{N} 2-\mathrm{C} 3-\mathrm{N} 6$ & $114.49(15)$ & $\mathrm{C} 17-\mathrm{O} 16-\mathrm{Fe} 1$ & 180 \\
\hline $\mathrm{C} 4-\mathrm{C} 3-\mathrm{N} 6$ & $122.40(17)$ & $\mathrm{O} 16-\mathrm{C} 17-\mathrm{C} 18$ & $121.51(12)$ \\
\hline $\mathrm{C} 5-\mathrm{C} 4-\mathrm{C} 3$ & $116.47(19)$ & $\mathrm{C} 18^{\mathrm{i}}-\mathrm{C} 17-\mathrm{C} 18$ & $117.0(2)$ \\
\hline $\mathrm{C} 5-\mathrm{C} 4-\mathrm{H} 4$ & 121.8 & $\mathrm{C} 17-\mathrm{C} 18-\mathrm{H} 18 \mathrm{~A}$ & 109.5 \\
\hline $\mathrm{C} 3-\mathrm{C} 4-\mathrm{H} 4$ & 121.8 & $\mathrm{C} 17-\mathrm{C} 18-\mathrm{H} 18 \mathrm{~B}$ & 109.5 \\
\hline $\mathrm{C} 4-\mathrm{C} 5-\mathrm{C} 4^{\mathrm{i}}$ & $121.9(2)$ & $\mathrm{H} 18 \mathrm{~A}-\mathrm{C} 18-\mathrm{H} 18 \mathrm{~B}$ & 109.5 \\
\hline $\mathrm{C} 4-\mathrm{C} 5-\mathrm{H} 5$ & 119.1 & $\mathrm{C} 17-\mathrm{C} 18-\mathrm{H} 18 \mathrm{C}$ & 109.5 \\
\hline $\mathrm{C} 10-\mathrm{N} 6-\mathrm{N} 7$ & $110.86(15)$ & $\mathrm{H} 18 \mathrm{~A}-\mathrm{C} 18-\mathrm{H} 18 \mathrm{C}$ & 109.5 \\
\hline $\mathrm{C} 10-\mathrm{N} 6-\mathrm{C} 3$ & $128.55(16)$ & $\mathrm{H} 18 \mathrm{~B}-\mathrm{C} 18-\mathrm{H} 18 \mathrm{C}$ & 109.5 \\
\hline $\mathrm{N} 7-\mathrm{N} 6-\mathrm{C} 3$ & $120.54(14)$ & $\mathrm{F} 22 \mathrm{~A}-\mathrm{B} 19 \mathrm{~A}-\mathrm{F} 20 \mathrm{~A}$ & $110.9(5)$ \\
\hline $\mathrm{C} 8-\mathrm{N} 7-\mathrm{N} 6$ & $105.11(14)$ & $\mathrm{F} 22 \mathrm{~A}-\mathrm{B} 19 \mathrm{~A}-\mathrm{F} 21 \mathrm{~A}$ & $110.1(4)$ \\
\hline $\mathrm{C} 8-\mathrm{N} 7-\mathrm{Fe} 1$ & $145.04(12)$ & $\mathrm{F} 20 \mathrm{~A}-\mathrm{B} 19 \mathrm{~A}-\mathrm{F} 21 \mathrm{~A}$ & $105.6(4)$ \\
\hline N6-N7-Fe1 & $109.72(10)$ & $\mathrm{F} 22 \mathrm{~A}-\mathrm{B} 19 \mathrm{~A}-\mathrm{F} 23 \mathrm{~A}$ & $113.5(5)$ \\
\hline $\mathrm{N} 7-\mathrm{C} 8-\mathrm{C} 9$ & $110.31(16)$ & $\mathrm{F} 20 \mathrm{~A}-\mathrm{B} 19 \mathrm{~A}-\mathrm{F} 23 \mathrm{~A}$ & $109.5(5)$ \\
\hline $\mathrm{N} 7-\mathrm{C} 8-\mathrm{C} 11$ & $122.06(16)$ & $\mathrm{F} 21 \mathrm{~A}-\mathrm{B} 19 \mathrm{~A}-\mathrm{F} 23 \mathrm{~A}$ & $107.0(5)$ \\
\hline $\mathrm{C} 9-\mathrm{C} 8-\mathrm{C} 11$ & $127.62(17)$ & $\mathrm{F} 23 \mathrm{~B}-\mathrm{B} 19 \mathrm{~B}-\mathrm{F} 21 \mathrm{~B}$ & $113.0(10)$ \\
\hline $\mathrm{C} 10-\mathrm{C} 9-\mathrm{C} 8$ & $106.30(17)$ & $\mathrm{F} 23 \mathrm{~B}-\mathrm{B} 19 \mathrm{~B}-\mathrm{F} 20 \mathrm{~B}$ & $110.9(10)$ \\
\hline $\mathrm{C} 10-\mathrm{C} 9-\mathrm{H} 9$ & 126.8 & $\mathrm{~F} 21 \mathrm{~B}-\mathrm{B} 19 \mathrm{~B}-\mathrm{F} 20 \mathrm{~B}$ & $112.3(10)$ \\
\hline $\mathrm{C} 8-\mathrm{C} 9-\mathrm{H} 9$ & 126.8 & $\mathrm{~F} 23 \mathrm{~B}-\mathrm{B} 19 \mathrm{~B}-\mathrm{F} 22 \mathrm{~B}$ & $103.3(10)$ \\
\hline $\mathrm{C} 9-\mathrm{C} 10-\mathrm{N} 6$ & $107.41(17)$ & $\mathrm{F} 21 \mathrm{~B}-\mathrm{B} 19 \mathrm{~B}-\mathrm{F} 22 \mathrm{~B}$ & $107.3(9)$ \\
\hline $\mathrm{C} 9-\mathrm{C} 10-\mathrm{H} 10$ & 126.3 & $\mathrm{~F} 20 \mathrm{~B}-\mathrm{B} 19 \mathrm{~B}-\mathrm{F} 22 \mathrm{~B}$ & $109.7(9)$ \\
\hline $\mathrm{N} 6-\mathrm{C} 10-\mathrm{H} 10$ & 126.3 & $\mathrm{O} 24-\mathrm{C} 25-\mathrm{C} 26$ & $121.6(2)$ \\
\hline $\mathrm{C} 8-\mathrm{C} 11-\mathrm{C} 14$ & $109.96(16)$ & $\mathrm{O} 24-\mathrm{C} 25-\mathrm{C} 27$ & $120.6(2)$ \\
\hline $\mathrm{C} 8-\mathrm{C} 11-\mathrm{C} 13$ & $109.10(15)$ & $\mathrm{C} 26-\mathrm{C} 25-\mathrm{C} 27$ & $117.8(2)$ \\
\hline $\mathrm{C} 14-\mathrm{C} 11-\mathrm{C} 13$ & $108.86(15)$ & $\mathrm{C} 25-\mathrm{C} 26-\mathrm{H} 26 \mathrm{~A}$ & 109.5 \\
\hline $\mathrm{C} 8-\mathrm{C} 11-\mathrm{C} 12$ & $109.79(15)$ & $\mathrm{C} 25-\mathrm{C} 26-\mathrm{H} 26 \mathrm{~B}$ & 109.5 \\
\hline $\mathrm{C} 14-\mathrm{C} 11-\mathrm{C} 12$ & $108.84(17)$ & $\mathrm{H} 26 \mathrm{~A}-\mathrm{C} 26-\mathrm{H} 26 \mathrm{~B}$ & 109.5 \\
\hline $\mathrm{C} 13-\mathrm{C} 11-\mathrm{C} 12$ & $110.28(16)$ & $\mathrm{C} 25-\mathrm{C} 26-\mathrm{H} 26 \mathrm{C}$ & 109.5 \\
\hline $\mathrm{C} 11-\mathrm{C} 12-\mathrm{H} 12 \mathrm{~A}$ & 109.5 & $\mathrm{H} 26 \mathrm{~A}-\mathrm{C} 26-\mathrm{H} 26 \mathrm{C}$ & 109.5 \\
\hline
\end{tabular}


supporting information

$\begin{array}{llll}\mathrm{C} 11-\mathrm{C} 12-\mathrm{H} 12 \mathrm{~B} & 109.5 & \mathrm{H} 26 \mathrm{~B}-\mathrm{C} 26-\mathrm{H} 26 \mathrm{C} & 109.5 \\ \mathrm{H} 12 \mathrm{~A}-\mathrm{C} 12-\mathrm{H} 12 \mathrm{~B} & 109.5 & \mathrm{C} 25-\mathrm{C} 27-\mathrm{H} 27 \mathrm{~A} & 109.5 \\ \mathrm{C} 11-\mathrm{C} 12-\mathrm{H} 12 \mathrm{C} & 109.5 & \mathrm{C} 25-\mathrm{C} 27-\mathrm{H} 27 \mathrm{~B} & 109.5 \\ \mathrm{H} 12 \mathrm{~A}-\mathrm{C} 12-\mathrm{H} 12 \mathrm{C} & 109.5 & \mathrm{H} 27 \mathrm{~A}-\mathrm{C} 27-\mathrm{H} 27 \mathrm{~B} & 109.5 \\ \mathrm{H} 12 \mathrm{~B}-\mathrm{C} 12-\mathrm{H} 12 \mathrm{C} & 109.5 & \mathrm{C} 25-\mathrm{C} 27-\mathrm{H} 27 \mathrm{C} & 109.5 \\ \mathrm{C} 11-\mathrm{C} 13-\mathrm{H} 13 \mathrm{~A} & 109.5 & \mathrm{H} 27 \mathrm{~A}-\mathrm{C} 27-\mathrm{H} 27 \mathrm{C} & 109.5 \\ \mathrm{C} 11-\mathrm{C} 13-\mathrm{H} 13 \mathrm{~B} & 109.5 & \mathrm{H} 27 \mathrm{~B}-\mathrm{C} 27-\mathrm{H} 27 \mathrm{C} & 109.5 \\ \mathrm{H} 13 \mathrm{~A}-\mathrm{C} 13-\mathrm{H} 13 \mathrm{~B} & 109.5 & & \end{array}$

Symmetry code: (i) $-x, y,-z+1 / 2$.

Hydrogen-bond geometry $\left(\AA,{ }^{\circ}\right)$

\begin{tabular}{lllll}
\hline$D-\mathrm{H} \cdots A$ & $D-\mathrm{H}$ & $\mathrm{H} \cdots A$ & $D \cdots A$ & $D-\mathrm{H} \cdots A$ \\
\hline $\mathrm{O} 15-\mathrm{H} 15 A \cdots \mathrm{F} 21 A$ & $0.80(3)$ & $1.99(3)$ & $2.771(3)$ & $164(3)$ \\
$\mathrm{O} 15-\mathrm{H} 15 A \cdots \mathrm{F} 20 B$ & $0.80(3)$ & $2.01(3)$ & $2.715(5)$ & $147(3)$ \\
$\mathrm{O} 15-\mathrm{H} 15 B \cdots \mathrm{O} 24$ & $0.79(3)$ & $1.92(3)$ & $2.689(2)$ & $165(3)$ \\
\hline
\end{tabular}

